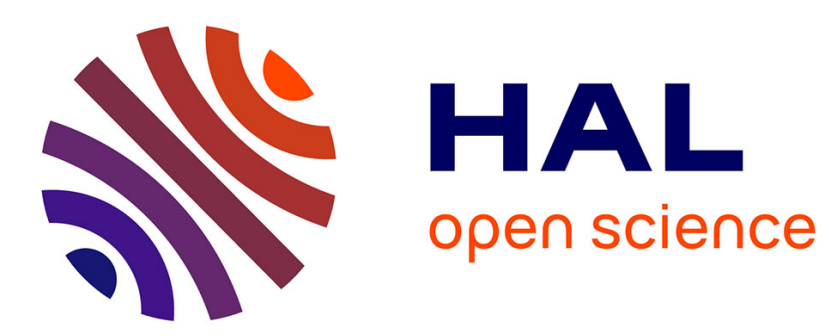

\title{
"Manger gras. Lard, saindoux, beurre et huile dans les traités de cuisine du Moyen Âge au XXe siècle" (2009)
}

Patrick Rambourg

\section{To cite this version:}

Patrick Rambourg. "Manger gras. Lard, saindoux, beurre et huile dans les traités de cuisine du Moyen Âge au XXe siècle" (2009). Représentations de l'obésité : le gros et le gras, May 2006, Paris, France. pp.75-91. hal-01138799

\section{HAL Id: hal-01138799 \\ https://hal.science/hal-01138799}

Submitted on 2 Apr 2015

HAL is a multi-disciplinary open access archive for the deposit and dissemination of scientific research documents, whether they are published or not. The documents may come from teaching and research institutions in France or abroad, or from public or private research centers.
L'archive ouverte pluridisciplinaire HAL, est destinée au dépôt et à la diffusion de documents scientifiques de niveau recherche, publiés ou non, émanant des établissements d'enseignement et de recherche français ou étrangers, des laboratoires publics ou privés. 


\title{
MANGER GRAS. LARD, SAINDOUX, BEURRE ET HUILE DANS LES TRAITÉS DE CUISINE DU MOYEN ÂGE AU XXE SIÈCLE
}

\author{
Patrick Rambourg \\ in Julia Csergo , Trop gros?
}

\section{Autrement | Mutations}

\section{9}

pages 75 à 91

Article disponible en ligne à l'adresse:

http://www.cairn.info/trop-gros---page-75.htm

Pour citer cet article :

Rambourg Patrick, «Manger gras. Lard, saindoux, beurre et huile dans les traités de cuisine du moyen âge au XXe siècle », in Julia Csergo, Trop gros ?

Autrement «Mutations », 2009 p. 75-91.

Distribution électronique Cairn.info pour Autrement.

(c) Autrement. Tous droits réservés pour tous pays.

La reproduction ou représentation de cet article, notamment par photocopie, n'est autorisée que dans les limites des conditions générales d'utilisation du site ou, le cas échéant, des conditions générales de la licence souscrite par votre établissement. Toute autre reproduction ou représentation, en tout ou partie, sous quelque forme et de quelque manière que ce soit, est interdite sauf accord préalable et écrit de l'éditeur, en dehors des cas prévus par la législation en vigueur en France. II est précisé que son stockage dans une base de données est également interdit. 
MANGER GRAS.

LARD, SAINDOUX, BEURRE ET HUILE DANS LES TRAITÉS

DE CUISINE DU MOYEN ÂGE AU XXE SIÈCLE

Patrick Rambourg

L'intérêt des historiens pour les graisses culinaires n'est pas nouveau. Sous l'impulsion de Lucien Febvre, une première enquête sous forme de questionnaire avait été lancée au milieu des années 1930 concernant «l'utilisation des graisses en France dans la cuisine rurale ${ }^{1}$ ». Le chercheur y voyait « une fixité assez remarquable [qui] a la solidité, un peu partout, des habitudes qui ne sont plus mises en question ${ }^{2} »$. On put dès lors établir, en cartographiant les données de l'enquête, les aires de répartition des principales graisses de cuisine. On observa deux zones de saindoux dans le Nord-Est et le Midi, et entre les deux une « coulée de beurre » qui recouvre la Bretagne, suit la vallée de la Loire jusque dans le Lyonnais, pour ensuite se perdre dans les Alpes où elle se retrouve en conflit avec le saindoux. L'huile d'olive règne sur le littoral méditerranéen et la Provence "jusqu'au contrefort des Alpes», mais remonte curieusement les vallées du Rhône et du Doubs où elle entre en concurrence avec le beurre et le saindoux. Quant à la graisse d'oie, elle se limite à l'Alsace et au Midi languedocien, mais pas de façon homogène. Et il y a aussi l'huile de noix que l'on utilise dans le Centre et l'Est du pays ${ }^{3}$.

1. Lucien Febvre, "Enquêtes et problèmes : les graisses de cuisine usuelles ", in Encyclopédie française, t. XIV, Paris, Larousse, 1954, p. 14, 44-1.

2. Jean-Jacques Hémardinquer, «Les graisses de cuisine en France. Essais de cartes », in Pour une histoire de l'alimentation, Cahier des Annales, $\mathrm{n}^{\circ} 28,1970$, p. 254-271. Article refondu à partir de Annales, no 16, 1961, p. 747-771.

3. Lucien Febvre, Encyclopédie française, op. cit., p. 14, 44-1 et 2. 
Cette géographie des graisses amène son lot de questions, notamment sur les zones constituées et la diffusion des matières grasses. Il serait en effet illusoire de penser qu'il en a toujours été ainsi. «Car il ne suffit pas qu'il y ait quelques oies dans une contrée, écrit Febvre, pour que la graisse d'oie puisse être utilisée pour la cuisine. Il en faut des troupeaux. » De même «que le beurre ne triomphe pas nécessairement dans les pays d'élevage bovin ${ }^{4} »$, etc. Pour compléter l'étude, une seconde enquête est lancée en 1942 «sur les usages conservés ou remémorés par les anciens »- l'année 1914 étant le plus souvent évoquée dans les témoignages, elle devient la date de référence. On remarque ainsi une Bretagne bien moins beurrière qu'il n'y paraît. Avant 1914, le beurre «n'était employé que rarement dans les landes, les vallées du Scorff et de la Vilaine» de la «Haute-Bretagne morbihannaise », alors qu'il semblait régner " plus au nord et sur toute la pénéplaine centrale ».

En réalité, précise Jean-Jacques Hémardinquer dans son article sur «Les graisses de cuisine en France. Essais de cartes ${ }^{5}$ » (1970), c'est uniquement le fait des riches et des villes, ou des bourgs particulièrement actifs. Mais après la Grande Guerre, la consommation du beurre se propage chez les ruraux et dans les populations modestes qui y voyaient le symbole d'une amélioration de leur niveau de vie. Cette affirmation du beurre est d'ailleurs confortée par la troisième étude de 1952, une enquête de consommation par ménage (d'après M. Lengellé), qui a également été cartographiée, afin de mieux visualiser, comme pour les précédentes enquêtes, les «frontières régionales » entre les trois grandes graisses (beurre, saindoux, huile) ${ }^{6}$.

Mais si ces enquêtes renseignent sur la répartition des graisses en France dans la première partie $\mathrm{du} \mathrm{xx}^{\mathrm{e}}$ siècle, elles restent bien silencieuses sur les périodes antérieures. Dans «Le goût et la nécessité : sur l'usage des graisses dans les cuisines d'Europe occidentale (XIV eXVIII $^{e}$ siècle) ${ }^{7}$ ", article publié en 1983, Jean-Louis Flandrin aborde différemment le sujet. La question des graisses de cuisine devient pour lui

4. Lucien Febvre, op. cit., p. 14, 44-1 et 2 .

5. Jean-Jacques Hémardinquer, «Les graisses de cuisine en France. Essais de cartes », op. cit., note 2 .

6. Toutes les enquêtes ont été cartographiées dans l'article de Jean-Jacques Hémardinquer, op. cit.

7. Jean-Louis Flandrin, «Le goût et la nécessité : sur l'usage des graisses dans les cuisines d'Europe occidentale XIV ${ }^{e}$-XVIII ${ }^{e}$ siècle », Annales ESC, $38^{\mathrm{e}}$ année, $\mathrm{n}^{\circ} 2$, mars-avril 1983, p. 369-401. 
un moyen «de savoir dans quelle mesure le goût d'un peuple a été modelé par ses pratiques alimentaires traditionnelles - elles-mêmes tributaires de contraintes diverses - et dans quelle mesure au contraire pratiques et goûts se sont opposés ». Pour l'historien, il faut distinguer dans le comportement alimentaire « la part du goût et la part de la nécessité », où des contraintes plus ou moins conscientes imposent de manger ce que nous ne mangerions pas de façon habituelle ou par dégoût.

Des contextes religieux, politiques, socioéconomiques, culturels et diététiques, sans oublier l'environnement naturel, influèrent indéniablement sur l'évolution des pratiques et des choix alimentaires; il en est de même des modes et des «tendances du goût ». Au cœur de ces processus il y a les graisses de cuisine, car elles participent à la fois à la technique culinaire et à l'assaisonnement, tout en ayant bien souvent une vocation identitaire. Les analyser sur la longue durée, grâce entre autres aux recueils de recettes, permet ainsi de constater que les matières grasses ne sont pas des aliments anodins. Elles jouèrent en effet un rôle important dans l'histoire de la cuisine comme dans les mutations du goût. Trois principaux corps gras occuperont le devant de la scène : le beurre, la graisse de porc (saindoux et lard) et l'huile. Leur statut variera selon les époques, le gras n'ayant pas toujours eu la mauvaise réputation que nous lui connaissons aujourd'hui ${ }^{8}$.

\section{La montée du beurre}

Dans Le Viandier ${ }^{9}$ imprimé de 1486, le sain, qui est une graisse située «entre les boyaulx et la haste menue » du porc, selon Le Mesnagier de Paris $^{10}$ (1393), devance largement le beurre et le lard ${ }^{11}$, l'huile n'étant employée que dans six recettes sur un corpus de 238 formules (histogramme $\mathrm{n}^{\circ} 1$ ). Près de trois quarts de siècle plus tard, le beurre est le

8. Claude Fischler consacre un chapitre à «la société lipophobe » dans L'Homnivore, Paris, Odile Jacob, 2001, p. 309-333.

9. Taillevent, Le Viandier, Houilles, Manucius, 2001. Nous avons volontairement commencé notre étude avec le premier livre de cuisine imprimé en France, sachant que les premiers manuscrits culinaires en langue vernaculaire apparaissent au tournant des XIII ${ }^{e}$ et XIV ${ }^{e}$ siècles.

10. Le Mesnagier de Paris, Paris, Livre de Poche, «Lettres gothiques », 1994, p. 596.

11. Le lard est une graisse ferme située entre la peau et la chair du porc. 


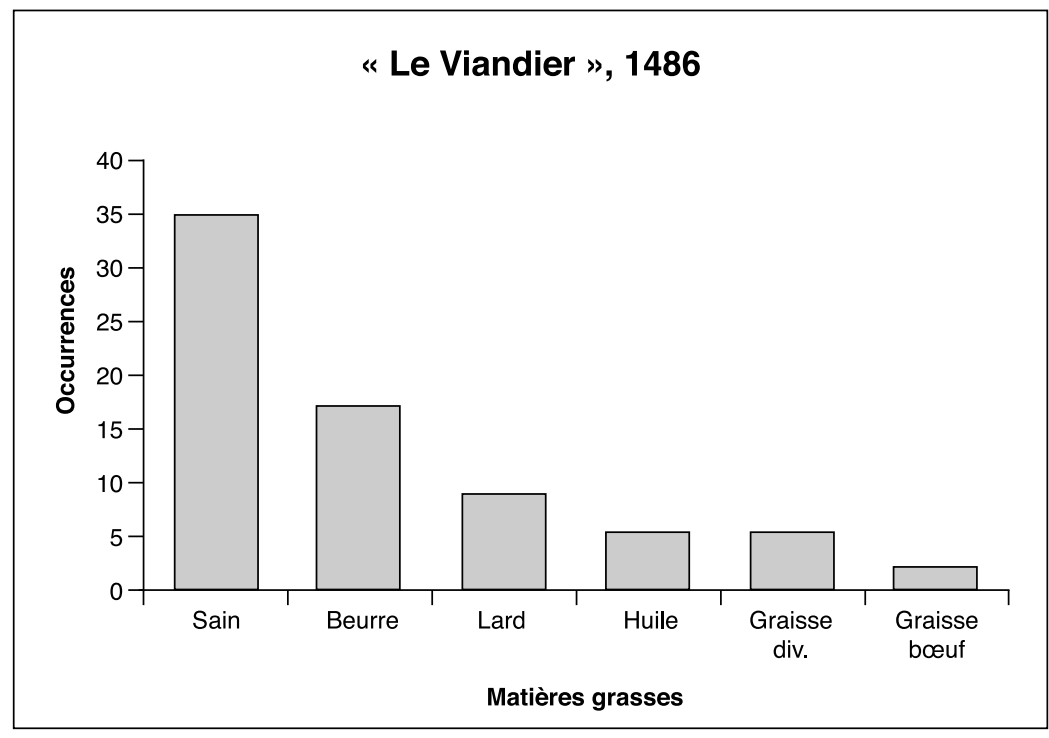

corps gras le plus utilisé dans Le Livre fort excellent de cuysine ${ }^{12}$ (1555), suivi du lard et du sain, l'huile n'arrivant que très loin derrière après la graisse de bœuf, et ne représentant qu'un peu plus de $7 \%$ des matières grasses relevées dans l'ouvrage (histogramme $\mathrm{n}^{\circ} 2$ ). À la Renaissance, le beurre fait une entrée remarquée dans les traités culinaires. Les références y sont nombreuses. Le Livre fort excellent de cuysine évoque ainsi le «beurre frais», le «bon beurre», le «bon beurre frais», le «beau beurre », ou encore le «beurre affiné » et le « beurre fondu», etc.

Le phénomène était déjà perceptible dans les recueils de recettes de la fin du $X^{e}$ siècle alors que dans les traités culinaires du siècle précédent, le beurre était quasi absent. Ce qui ne veut pas dire pour autant que l'on n'en consommait pas. Vers 1451, Gilles Le Bouvier, dit Berry, héraut d'armes de Charles VII, constatait ainsi, dans son Livre de la description des pays, que les Bretons « font moult de beurre, qu'ils vendent aux pays étrangers et mangent en carême faute $d^{\prime}$ huile $^{13}{ }$. Les comptes

12. Le Livre fort excellent de cuysine, Lyon, 1555.

13. Gilles Le Bouvier, dit Berry, Le Livre de la description des pays, Paris, Ernest Leroux, 1908, p. 49. 


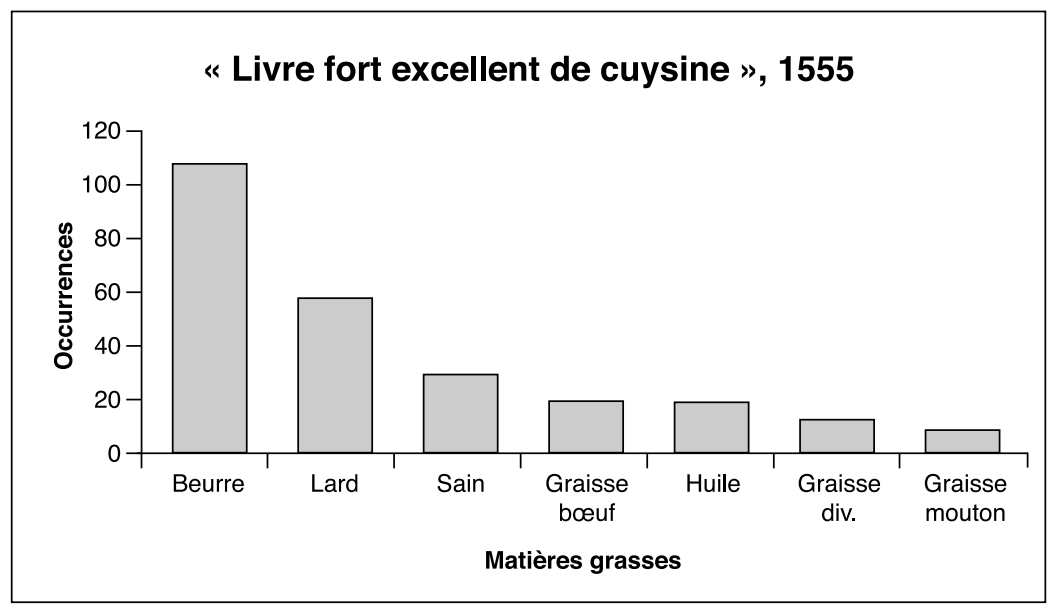

de Jean d'Angoulême $(1462)^{14}$, qui résidait à Cognac, témoignent également de l'achat de grandes quantités de beurre et de son utilisation culinaire les vendredis, samedis et mercredis ; c'est-à-dire des jours où le chrétien ne pouvait manger de viande ${ }^{15}$. Car l'Église, qui interdisait la consommation des matières grasses animales durant le carême, mais aussi pendant les jours maigres, avait fini par autoriser la consommation du beurre, notamment dans les régions «beurrières » où l'huile était rare et chère.

Cette dispense s'adressait d'abord aux moins fortunés, le beurre étant alors considéré comme «la graisse des pauvres ${ }^{16}$ ", ce qui expliquerait sa rareté dans les recueils de recettes médiévaux qui présentaient avant tout une cuisine d'élite ${ }^{17}$. Jean Nicot écrira encore au début du

14. Jean-Louis Flandrin, «Et le beurre conquit la France », L'Histoire, ${ }^{\circ} 85$, janvier 1986, p. 108-111.

15. Patrick Rambourg, «L'Abbaye de Saint-Amand de Rouen (1551-1552) : de la différenciation sociale des consommateurs, au travers des aliments, à la pratique culinaire ", in Production alimentaire et lieux de consommation dans les établissements religieux au Moyen Âge et à l'époque moderne, actes du colloque de Lille, 16-19 oct. 2003, Benoît Clavel (dir.), Histoire médiévale et archéologie, t. I, n 19 , 2006, p. 217-229.

16. Jean-Louis Flandrin, «Et le beurre conquit la France», op. cit., p. 110.

17. Patrick Rambourg, De la cuisine à la gastronomie : histoire de la table française, Paris, Louis Audibert, 2005, p. 84. 
XVII siècle, dans son Thresor de la langue francoyse, que le beurre en France est d'un usage commun, mais «plus du pauvre que du riche ${ }^{18}$ ». La dispense de l'Église va jouer un rôle important dans l'évolution de ce corps gras, que l'on peut désormais utiliser quotidiennement, et qui trouvera peu à peu sa place dans les traités culinaires. Ainsi dans Le Viandier de 1486 , le beurre est-il souvent conseillé dans les recettes "maigres ${ }^{19}$ ", en remplacement d'une autre matière grasse ; à l'exemple de la "porée » (un hachis de légumes généralement verts), où la "purée de pois » (une sorte de bouillon) et le beurre se substituent au lard, au sain et au bouillon de bœuf lorsque le plat est apprêté un « jour à poisson ». Dans d'autres recettes, il est l'équivalent de l'huile, telle la « soupe à la moutarde » où les œufs peuvent être frits au beurre ou à l'huile.

Si les prescriptions religieuses influèrent sur l'emploi et le choix des graisses en cuisine, le goût n'en était pas oublié pour autant. Le beurre de Vanves, dans la région parisienne, est ainsi recherché pour «son parfum et sa saveur ». Celui des montagnes du Lyonnais est "très savoureux et toujours d'une belle couleur», et les habitants de Blois sont fiers de leur beurre, écrit Bruyerin-Champier $(1560)^{20}$, le médecin de François I ${ }^{\text {er }}$. À la même époque, la Bretagne et la Normandie sont déjà de grandes productrices de beurre (salé) qu'elles transportent dans des pots de terre vers les autres provinces ou à l'étranger ${ }^{21}$. Et à l'aube du XVII siècle, l'agronome Olivier de Serres conseille le beurre «du mois de mai à tout autre, pour sa belle couleur dorée et grande délicatesse ${ }^{22}$ », et plus généralement les beurres produits au printemps et à l'automne qui sont meilleurs et en plus grande abondance que ceux de l'été et de l'hiver. Il propose également une façon de le conserver longuement tout en le gardant bon :

Comment le garder bon. Pour longuement conserver le beurre, ne faut que le saler: mais beaucoup plus délicat se rend-il par le

18. Thresor de la langue francoyse tant ancienne que moderne..., Paris, 1606.

19. Les recettes des livres de cuisine seront longtemps classées suivant les jours gras et les jours maigres.

20. De re cibaria, traduit par Sigurd Amundsen, Paris, L'intermédiaire des chercheurs et curieux, 1998, p. 440.

21. Fabienne Ravoire, "Le voyage des pots de beurre », in Jean-Louis Flandrin et Carole Lambert (dir.), Fêtes gourmandes au Moyen Âge, Paris, Imprimerie nationale, 1998, p. 140.

22. Olivier de Serres, Le Théâtre d'agriculture et Mesnage des champs (1600), Arles, Actes Sud, 1996, p. 423. 
bouillir, tellement qu'ainsi accommodé, peu de différence y a-t-il de lui au récent et frès. En le bouillant sur feu clair et lent, le faut escumer, pour le descharger curieusement, de tout le terrestre qu'en se cuisant il expulse en haut: dont en fin il se représente clair et de couleur blonde, comme beau huile d'olive, puis mis dans des vazes de terre vitrés au dedans, est gardé tant longuement qu'on désire; ainsi le pratique-on en Lorraine ${ }^{23}$.

\section{Entre beurre et lard}

Cette progression régulière du beurre dans les livres de cuisine traduit une mutation du goût qui tend vers plus de douceur par rapport au goût épicé et acide de la période médiévale. Cette évolution se constate également dans l'utilisation croissante du sucre dans les préparations culinaires ${ }^{24}$. Le beurre devient peu à peu la graisse de référence de la cuisine française, à un moment où celle-ci conforte sa réputation. Dans Le Cuisinier $^{25}$ de Pierre de Lune, publié en 1656, le beurre apparaît dans au moins $37 \%$ des recettes, alors que le saindoux, qui était la première graisse du Viandier imprimé, ne figure que dans un peu plus de $1 \%$ des recettes, soit une douzaine seulement (histogramme $n^{\circ} 3$ ). Dans l'ouvrage, le beurre est principalement employé dans la préparation des poissons et des légumes : cela n'a rien de surprenant puisqu'il a d'abord été perçu comme une graisse des jours maigres. On le retrouve aussi dans nombre de pâtisseries, notamment dans la confection de la pâte. Il s'affirme surtout dans les sauces, l'apparition des sauces au beurre allant de pair avec l'essor du beurre dans les livres de cuisine ${ }^{26}$.

La «sauce blanche » et le «beurre blanc » se confectionnent ainsi avec des ingrédients acides (vinaigre, vin blanc, verjus) que l'on mélange à du beurre, à l'aide d'une cuillère, « sans discontinuer, afin que la sauce

23. Olivier de Serres, Le Théâtre d'agriculture et Mesnage des champs, op.cit., p. 423-424.

24. Patrick Rambourg, «Sucre et mets sucrés dans les traités culinaires de la fin du Moyen Âge au début du Xxe siècle, in Du sucre, actes de la journée d'études «Le sucre dans la littérature »(Pau, 21 janvier 2005), Biarritz, Atlantica, 2007, p. 173-188.

25. L'Art de la cuisine française au XVII siècle, Paris, Payot \& Rivages, 1995, p. 239-437.

26. Patrick Rambourg, De la cuisine à la gastronomie..., op. cit., p. 101. 
se lie et devienne bien épaisse ", explique un auteur de l'époque ${ }^{27}$. La « sauce rousse » ressemble davantage à un beurre fondu roux dans lequel on incorpore, entre autres, du persil haché et des câpres... D'une façon générale, on prendra l'habitude de terminer les sauces avec « un petit morceau de beurre manié pour donner un corps ». Mais «il faut que le peu de beurre qui entre dans toutes ces sauces soit parfait pour la qualité, autrement il vaudrait mieux s'en passer $"$, dit un chef des Lumières ${ }^{28}$. Le beurre devient également la graisse de prédilection du roux - cette nouvelle technique de liaison apparue au cours du Grand Siècle qui remplacera la pratique médiévale au pain.

Deux sauces pour les darnes de saumon frais. L'une blanche avec beurre frais, verjus, citron, orange, poivre, sel menu, un anchois battu; délayer le tout ensemble et y ajouter une cuillerée de liaisons ou jus d'écrevisses s'il y en a, et verser chaudement dessus. L'autre rousse, qui se fait ainsi : faites fondre du beurre frais, et quand il sera roux, jetez dedans du persil haché menu, deux ou trois anchois ; préparez peu de bouillon de poisson ou de purée sans herbes, quelque jus ou liaison, câpres, sel, épices, une cuillerée de verjus et moitié autant de vinaigre; faites bien consommer cette sauce, en sorte qu'elle devienne toute en liaison, et la versez sur vos darnes aussitôt pour donner à manger chaudement, car de telles sauces réchauffées tournent facilement en huile ${ }^{29}$.

Mais le beurre n'est pas le seul corps gras en progression ; le lard est également de plus en plus employé. Il l'est dans près de $29 \%$ des recettes de Pierre de Lune, et représente presque $32 \%$ des graisses répertoriées dans la Suite des Dons de Comus de François Marin, édité en 1742 (histogramme $n^{\circ} 4$ ). Le lard « est proprement ce qui est compris entre l'épaule et la cuisse du porc, depuis l'échine jusqu'au-dessous du ventre des deux côtés », explique, à la fin des années 1760, le Dictionnaire portatif de cuisine ${ }^{30}$. Il est utilisé sous diverses formes, principalement dans

27. L. S. R., L'Art de bien traiter, 1674, in L'Art de la cuisine française au XVII siècle, op. cit., p. 139.

28. François Marin, Suite des Dons de Comus, t. I (Paris, 1742), Pau, Manucius, 2001, p. 53.

29. L. S. R., L'Art de bien traiter, op. cit., p. 141.

30. Dictionnaire portatif de cuisine (1767), Paris, Payot \& Rivages, 1995, p. 257. 


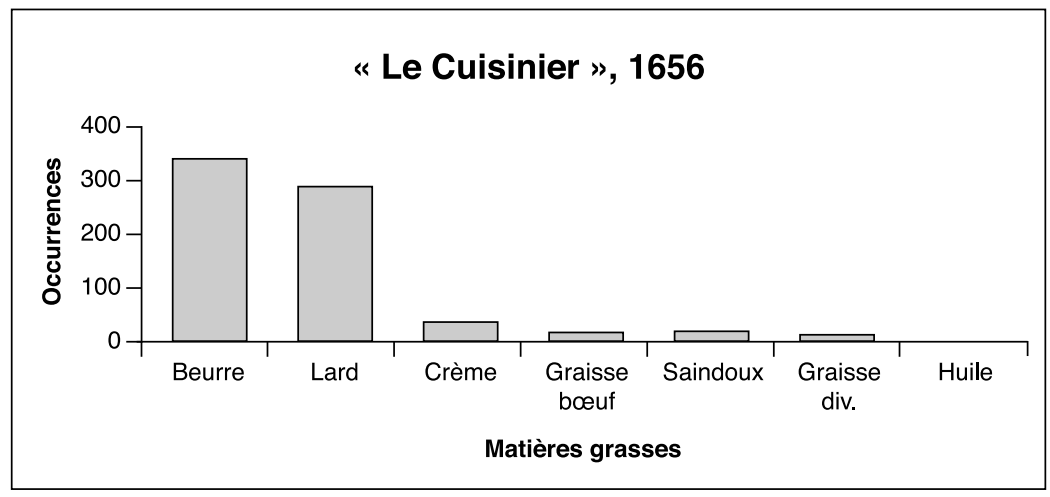

l'apprêtement des viandes : on le fait fondre pour en faire revenir les morceaux afin qu'ils prennent une belle « couleur blonde ${ }^{31}$ ». Le «petit lard» est « un morceau de cochon où il y a de la chair qui tient à la graisse »; l'« entrelardé » est considéré comme le meilleur et le plus appétissant, car «la graisse et la chair se trouvent distribuées en plusieurs couches ». La «flèche de lard » est la graisse "qu'on lève tout le long d'un des côtés du porc qu'on sale et qu'on garde longtemps ${ }^{32}{ }^{\text {» }}$.

Façon de faire le lard. Il faut lever le lard de dessus le porc \& y laisser le moins de chair qu'on pourra. Ayez du sel pilé. Salez votre lard \& arrangez le bien sur des planches dans la cave, mettez-y encore du sel tout autour \& par-dessus. Mettez les morceaux l'un sur l'autre, chair contre chair. Il faut une livre \& demie de sel sur vingt livres de lard, laissez-le dans le sel le plus que vous pourrez. Ensuite vous le suspendez dans un endroit sec pour le sécher, \& qu'il se fasse bien $^{33}$.

Le lard gras sert surtout à larder et barder les pièces de viande à rôtir. La première technique consiste à introduire des bâtonnets de lard

31. «Si la couleur ne prenoit pas, il faudroit y jetter un peu de lard fondu ou autre graisse fonduë. Cela fait revenir la couleur blonde en général, sur tout ce qui est pané », in François Marin, Suite des Dons de Comus, op. cit., t. I, p. 296.

32. Dictionnaire portatif de cuisine, op. cit., p. 257.

33. François Marin, Suite des Dons de Comus, op. cit., t. II, p. 9-10. 
plus ou moins gros dans la chair pour la nourrir durant sa cuisson et éviter son dessèchement. Avec la seconde technique, la viande est protégée d'une tranche de lard que l'on ficelle dessus. Rappelons que le rôtissage des viandes se faisait à l'âtre de la cheminée, devant un feu plus ou moins contrôlé. Nombre de tableaux de l'époque témoignent de ces pratiques culinaires, représentatives de la cuisine des XVI' et XVIII siècles, comme la Venaison piquée pour mise en broche (1716) d'Alexandre-François Desportes, une commande du Régent pour sa cuisine personnelle du Palais-Royal ${ }^{34}$, qui montre des volatiles lardés et bardés prêts à être cuits.

\section{Histogramme $n^{\circ} 4$}

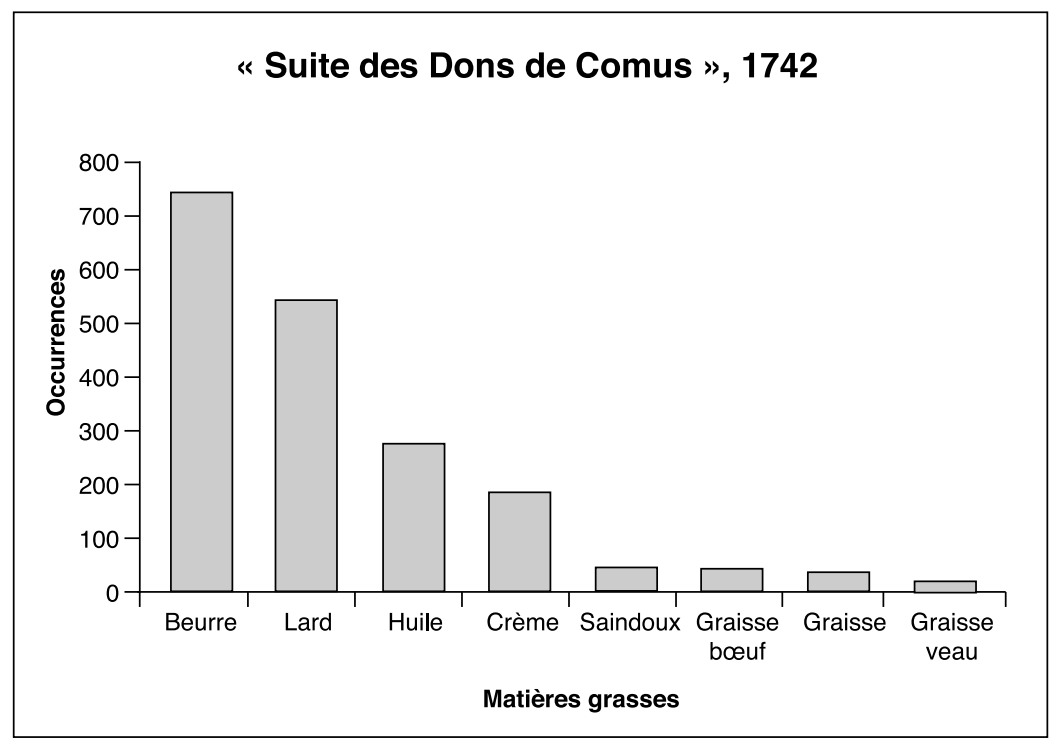

\section{Et l'huile !}

Ainsi le beurre et le lard sont-ils les corps gras les plus employés de la cuisine des Lumières, avec néanmoins des rôles bien distincts : le premier accompagne principalement les poissons et les plats de légumes, et sert bien souvent à faire revenir les volatiles lorsqu'on les prépare en

34. Livres en bouche, cinq siècles d'art culinaire français, Paris, Bibliothèque nationale de France et Hermann, 2001, p. 178. 
fricassée ; le second est surtout utilisé dans l'apprêt des pièces à rôtir, mais aussi dans les tourtes à base de viande... Quant à l'huile, elle se fait rare dans les livres de cuisine du Grand Siècle. Dans Le Cuisinier de Pierre de Lune (1656), elle apparaît dans les « pigeonneaux à l'italienne » où les oiseaux sont «trempés » et frits dans de l'huile d'olive. La référence à l'Italie n'est pas anodine : l'huile d'olive caractérise le monde méditerranéen. Le prêtre Jean-Jacques Bouchard qui, en 1630, partit de Paris pour un long voyage vers Rome, nota dans son journal qu'en Provence où « se ressent si fort [le] voisinage de l'Italie », le beurre est rarement utilisé, au bénéfice de l'huile qui accommode les poissons «tant en friture qu'en sauce, comme [...] les potages ${ }^{35} \ldots$ »

Mais si la partie septentrionale du pays use du beurre, alors que le Sud utilise l'huile d'olive, "les uns et les autres cuisinaient la majeure partie de l'année au lard, au saindoux ou avec d'autres graisses animales », écrit Jean-Louis Flandrin ${ }^{36}$. La rareté de l'huile dans le livre de Pierre de Lune ne signifie nullement qu'on n'en consommait pas au nord de la Loire, et particulièrement dans la capitale. Comme en témoigne l'ambassadeur vénitien Jérôme Lippomano dans les années 1570 : «L'huile a une saveur âcre; c'est pourquoi on apprête avec le beurre presque toutes sortes de mets, excepté la salade. La plus grande quantité d'huile vient de la Provence, et ce sont les épiciers qui la vendent ${ }^{37}$. » Un siècle plus tard, L. S. R., auteur anonyme de L'Art de bien traiter (1674), conseille d'avoir en cave « de la meilleure huile, de Nice, d'Aremont et autres lieux renommés de Provence, en pièce ou en fioles, il n'importe. Les bons magasins que nous en avons à Paris chez ces fameux épiciers des Halles nous doivent épargner la peine d'en faire venir de $\operatorname{si~loin~}^{38}$... » D'ailleurs L. S. R. utilise de la «bonne huile d'olive » mélangée à $d u$ «bon beurre frais » affiné, c'est-à-dire débarrassé de ses impuretés, pour faire ses fritures de poissons (entre autres).

Dans les recueils de recettes, l'huile progresse peu à peu. Elle arrive même en troisième position dans la Suite des Dons de Comus (1742), où elle représente plus de $16 \%$ des graisses répertoriées. Elle est

35. "Voyage de Paris à Rome », in Emanuele Kanceff (dir.), Euvres de Jean-Jacques Bouchard, Turin, G. Giappichelli, 1976-1977, vol. I, p. 76-80.

36. Jean-Louis Flandrin, "Le goût et la nécessité : sur l'usage des graisses... », op. cit., p. 380.

37. M. N. Tommaseo, Relations des ambassadeurs vénitiens sur les affaires de France au XVI siècle, Paris, Imprimerie royale, 1838, t. II, p. 575.

38. L. S. R., L'Art de bien traiter, op. cit., p. 80. 
généralement utilisée dans les fritures et les marinades, à l'exemple du «filet de bœuf à la Morguienne » que l'on marine «dans de l'huile trèsfine » avec des tranches de citron. Elle est également employée dans la conservation des aliments (artichauts, concombres, etc.), et sert à badigeonner des viandes, tel le cochon de lait avant son rôtissage. On la retrouve aussi dans les recettes se référant au Sud, «à l'espagnole", «à l'italienne », «à la provençale ${ }^{39}$ ", mais aussi dans nombre de sauces où elle est mélangée avec du beurre: à l'exemple de la «sauce au Monarque » composée d'un verre d'huile et de trois pains de beurre. Mais au cours du siècle suivant, l'huile se fera plus rare dans les sauces chaudes, au bénéfice du beurre, et s'imposera surtout dans les préparations froides dont les plus connues sont la mayonnaise et la vinaigrette.

\section{Le beurre : I'incontournable corps gras}

Des trois principales graisses que nous venons d'analyser, le beurre devient l'incontournable corps gras de la grande cuisine française du XIXe siècle et d'une bonne partie du XXe siècle. Dans Le Livre de cuisine $e^{40}$ de Jules Gouffé (1867), il apparaît dans plus de $72 \%$ des recettes utilisant les trois catégories de graisse (histogramme $\mathrm{n}^{\circ} 5$ ). Son usage est quasi général dans les plats de l'époque. Le morceau de beurre frais que l'on ajoute à la fin de la préparation culinaire est désormais la norme, et constitue pour Gouffé «le goût et la saveur de la sauce». Il doit cependant être de "première fraîcheur et de première qualité ». Toutefois certains pensent que le beurre «employé pour le roux n'a pas besoin d'être très frais ni très bon, sous prétexte qu'il est destiné à être cuit $^{41}{ }^{1}$. Gouffé récuse ce préjugé car «le mauvais beurre ne [...] donnera jamais que de mauvaises choses ». Le beurre trouvera également sa place sur la table, servi «en pain ou en coquille, toujours avec de l'eau très fraîche, sans trop remplir le bateau, pour éviter de renverser en faisant circuler ${ }^{42}$ ».

39. Patrick Rambourg, «L'appellation “à la provençale” dans les traités culinaires français du XVII e au XXe siècle", in Provence historique. Alimentation et cuisine en Provence, t. LIV, fascicule 218, octobre-décembre 2004, p. 473-483.

40. Jules Gouffé, Le Livre de cuisine (1867), Paris, Parangon, 2001.

41. Ibid., p. 301.

42. Ibid., p. 92. 
Le corps gras se fera encore plus visible avec la mode des beurres composés. Dans son chapitre «beurre pour sauces et garnitures", Jules Gouffé en propose sept recettes, dont le bien connu «beurre maître $\mathrm{d}^{\prime}$ hôtel $^{43}$ ", qui rencontrera le succès en accompagnement du steak ${ }^{44}$. Quelques décennies plus tard, Auguste Escoffier n'en présentera pas moins de 36 formules dans son Guide culinaire (1921), allant du «beurre d'ail » au «beurre de homard», en passant par le «beurre de caviar» et le «beurre de moutarde ». "Les beurres composés sont généralement préparés au moment ; s'ils le sont à l'avance, explique le grand chef, on doit les tenir au frais, et couverts d'un rond de papier blanc. » L'emploi de ces divers beurres dans les légumes, potages ou sauces, accentue et fixe «d'une façon définitive, la saveur propre et particulière de ces préparations ${ }^{45}$ ». Pour Escoffier, le beurre est un absorbeur de «particules savoriques et aromatiques » qu'il diffuse, en fondant dans la composition dans laquelle il a été mis.

\section{Histogramme $n^{\circ} 5$}

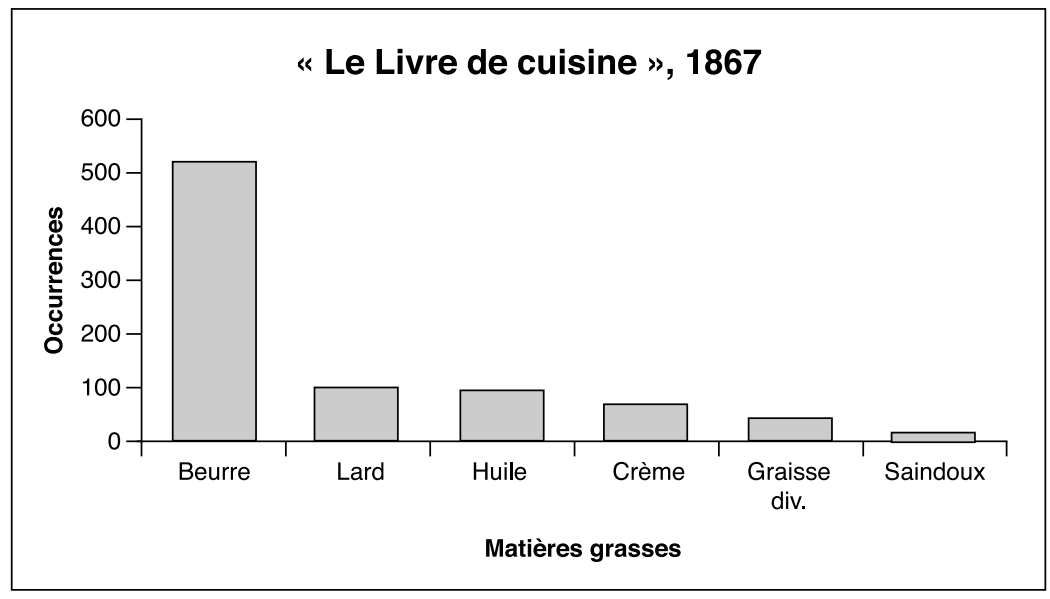

Au-delà des préférences gustatives, la plus grande utilisation du beurre, et du même coup, l'abandon progressif du lard ne sont pas sans

43. On mélange au beurre des fines herbes, généralement du persil haché, du citron, du sel et du poivre.

44. Patrick Rambourg, «Steak en sauce : ou comment les sauces françaises contribuèrent au succès du steak anglais aux $18^{\mathrm{e}}$ et $20^{\mathrm{e}}$ siècles », Papilles, $\mathrm{n}^{\circ} 25$, Bibliothèques gourmandes, éditions Virgile, novembre 2004, p. 29-43.

45. Auguste Escoffier, Le Guide culinaire (1921), Paris, Flammarion, 2001, p. 53. 
lien avec l'évolution technique des cuisines et des manières de cuisiner. L'affirmation du «potager » au XVII siècle contribuait déjà à une sophistication des pratiques culinaires. Cette table de maçonnerie, à hauteur d'appui, permettait de cuire en même temps diverses préparations sur des cavités remplies de braise et à l'ouverture plus ou moins grande ; le cuisinier pouvait ainsi avoir un meilleur contrôle des cuissons ${ }^{46}$, sachant que le beurre brûle rapidement sous l'effet d'une chaleur intense. Avec le XIXe siècle, le fourneau remplace peu à peu le "potager » et la cheminée s'efface progressivement. Le foyer du fourneau chauffe la plaque de cuisson placée au-dessus et les fours situés dans la partie inférieure. Le feu domestiqué rend moins utile l'emploi du lard qui préservait la viande du dessèchement. Certes des pièces comme le «filet de bœuf rôti » de Jules Gouffé sont encore lardées, mais l'utilisation du papier graissé couvrant la viande mise en broche (rôtisserie mécanique) devient habituelle.

Si la graisse de porc, dans ses variantes diverses (lard, saindoux), est de moins en moins employée dans l'apprêtement des aliments, elle est en revanche d'un grand usage dans la confection des socles et des piédestaux. Car la grande cuisine du XIXe siècle, du moins dans les premières décennies, est une cuisine de socles sur lesquels sont disposés les mets. Signe d'un nouvel esthétisme culinaire, Antonin Carême donnait l'exemple avec sa cuisine architecturale et ses fameux buffets pyramidaux ${ }^{47}$. Les socles sont faits en saindoux ou avec d'autres graisses plus ou moins fermes, mais toujours blanches et inodores, puis travaillés de manière à leur donner du corps, l'élasticité du produit permettant ainsi toutes les compositions ${ }^{48}$. On en confectionnait aussi pour garnir le fond des plats, ce qui avait parfois pour inconvénient de « répandre un certain goût de graisse ${ }^{49}$ ». Pour pallier ce problème, Jules Gouffé conseillait de la recouvrir d'une pâte de riz, elle-même couverte d'un beurre de Montpellier ou de ravigote ${ }^{50}$. Les corps gras sont ici omniprésents, mais jouent un rôle différent comme éléments de décoration, contribuant ainsi au prestige de la «cuisine décorative».

46. Patrick Rambourg, De la cuisine à la gastronomie..., op. cit., p. 110.

47. Ibid., p. 177.

48. Joseph Favre, Dictionnaire universel de cuisine pratique, Paris, seconde édition (vers 1905). Marseille, Laffitte reprints, 1978, t. III, p. 1079.

49. Jules Gouffé, Le Livre de cuisine, op. cit., p. 510.

50. Le premier était fait d'un mélange de cerfeuil, estragon, pimprenelle, civette, cresson, jaune d'œuf, anchois, cornichons, câpres et huile, le second était sensiblement de même composition mais sans les cinq derniers ingrédients. 


\section{À la recherche de la légèreté}

Au tournant des XIX et $X^{e}$ siècles, la cuisine de socles est cependant peu à peu abandonnée. Elle est devenue trop compliquée et trop longue à préparer, pour une clientèle toujours plus pressée. Dans la décoration des mets, on rejette tout ce qui n'est pas comestible : le saindoux disparaît progressivement des livres de cuisine. Quant au beurre, il reste la référence absolue jusqu'aux dernières décennies du $\mathrm{xx}^{\mathrm{e}}$ siècle. C'est encore vrai dans La Cuisine du marché de Paul Bocuse éditée en 1976. Mais La Grande Cuisine minceur ${ }^{51}$ de Michel Guérard, publiée la même année, offre une tout autre approche de la cuisine. Le beurre n'apparaît que dans $6 \%$ (environ) des « recettes minceur» (principalement dans la confection des pâtisseries), alors que l'huile est utilisée dans plus de $35 \%$ des recettes. Le livre rencontre un réel succès en déculpabilisant le plaisir de manger, et en alliant grande cuisine et diététique.

\section{Histogramme $n^{\circ} 6$}

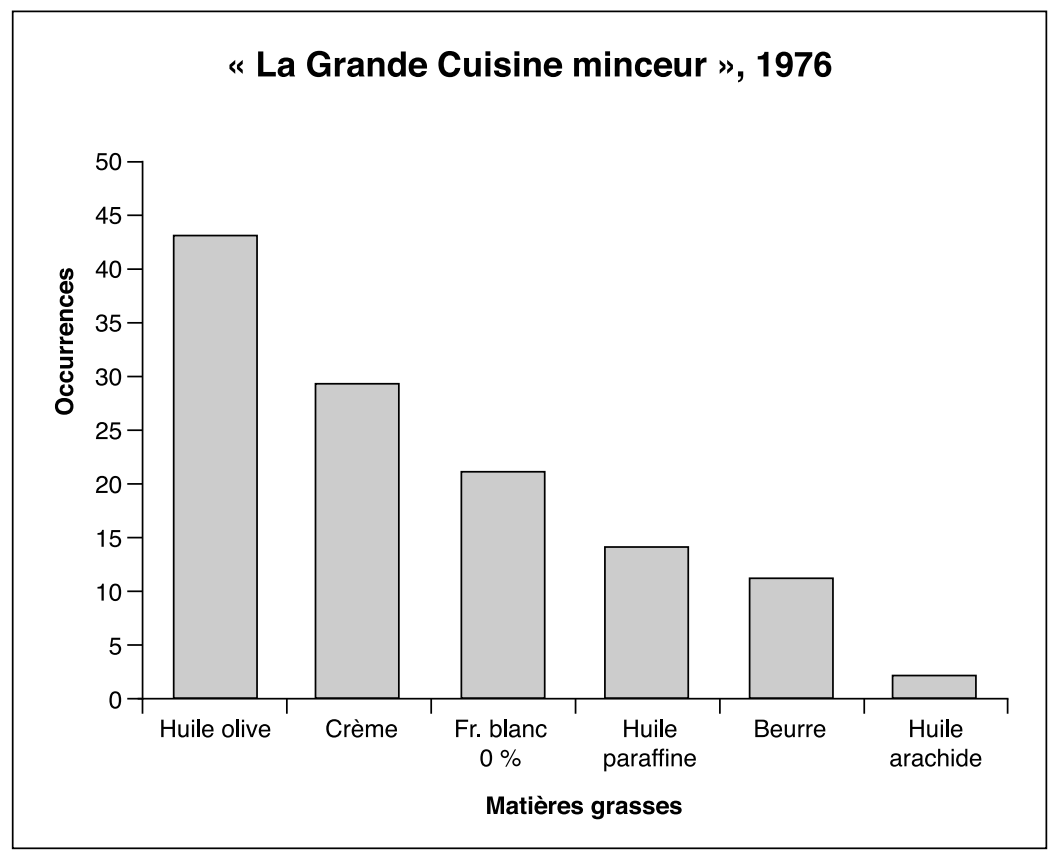

51. Michel Guérard, La Grande Cuisine minceur, Paris, Robert Laffont, 1976. 
Le contexte de l'époque et le culte du corps favorisent cette approche culinaire. La "nouvelle cuisine », qui est alors en vogue, prône la simplicité, le respect des saveurs et les principes diététiques ${ }^{52}$. Pour André Guillot, La Vraie Cuisine légère (1981) est savoureuse et "diététique par nature : pas de corps gras (ou très peu), pas de farine, pas de roux, pas de mixtures indigestes. De grands plats pour la dégustation, mais des plats simples dans leur conception, et qui ne laissent pas de lendemains qui déchantent ${ }^{53}$. » Les corps gras sont perçus comme superflus et nuisibles. "On a trop longtemps identifié la bonne cuisine aux matières grasses qu'elle utilisait, dit-il. Et pourtant, le gras est l'ennemi de la santé. » Pour lui, une belle sole cuite « sur des algues, sur des pierres chauffées ou un barbecue, ou bien à la vapeur d'un bouillon parfumé d'aromates et d'herbes ", a autant de saveur qu'une sole au beurre noisette. C'est une révolution! Le beurre, qui faisait le succès de la cuisine française, devient l'ennemi à abattre.

Cette recherche de légèreté se traduit dans l'évolution et la conception des sauces, où le roux est de plus en plus banni, car il leur donne une sapidité pâteuse, et les rend grasses. Mais en divisant par deux la quantité de beurre entrant dans la confection du roux, on obtient une sauce «lipidiquement » plus légère, explique Guérard. Il suggère aussi la liaison à l'amidon qui est « hygiéniquement parlant plus [légère] à la digestion qu'une sauce nourrie outrancièrement de beurre et de crème réduite ${ }^{54} »$. Mais il préfère, pour sa cuisine minceur, utiliser des yaourts et des fromages blancs à $0 \%$ de matière grasse, de la crème fraîche à $20 \%$ et des purées de légumes qui apportent finesse et onctuosité grâce au mixer à grande vitesse. La réduction du fond de sauce est également une autre façon de procéder ; elle permet un épaississement du liquide, sans emploi, ou presque, de matière grasse.

Pourtant la notion de légèreté n'est pas nouvelle, mais sa signification a évolué selon les époques. Face aux graisses, les cuisiniers ont très tôt pris l'habitude de dégraisser leurs sauces et leurs potages. En 1486, Le Viandier conseillait déjà de retirer la graisse que l'on voyait trop dans la recette des "poussins à l'étuvée ». Pierre de Lune, un peu moins de deux siècles plus tard, suggérait de dégraisser la sauce du «membre de mouton à la ficelle» et les "poulets fricassés à la crème», dont les

52. Patrick Rambourg, De la cuisine à la gastronomie..., op. cit., p. 233.

53. André Guillot, La Vraie Cuisine légère, Paris, Flammarion, 1993, p. 5.

54. Michel Guérard, La Grande Cuisine minceur, op. cit., p. 73-74. 
morceaux avaient été préalablement passés à la poêle dans du lard, et cuits avec « un peu de bon beurre», avant d'être crémés.

Dans son Art de bien traiter (1674), L. S. R. observe que la chair la moins grasse est « toujours la meilleure » pour le bouillon, « et qu'il n'a rien de plus insupportable et de plus dégoûtant que de voir surnager pour ainsi dire de l'huile sur des potages et autres assaisonnements, qui sont capables de nous ôter l'appétit et provoquer même le vomissement ${ }^{55}$ ». Le terme «dégraissé » devient d'un emploi courant dans la langue des cuisiniers : il est employé maintes fois dans la Suite des Dons de Comus (1742). La sauce à la carpe est « légère » lorsqu'on la dégraisse bien, dit Marin. À cette époque, la légèreté recherchée correspond avant tout à une réduction des quantités de matières grasses utilisées. Pour confectionner la «sauce à l'italienne » il faut prendre «deux pains de beurre » et un verre d'huile, alors que la même sauce qualifiée de «légère » n'aura $\mathrm{qu}^{\prime}$ « un ou deux pains de beurre » et un demi-verre d'huile. Ainsi les cuisiniers ont-ils très tôt fait le lien entre la diminution des corps gras et la notion de légèreté.

Dans cette histoire des corps gras, l'inexorable ascension du beurre, commencée à la Renaissance, s'interrompt dans les dernières décennies du Xxe siècle. Il n'est ainsi plus considéré comme la graisse de référence de la cuisine française. C'est désormais un ingrédient que l'on consomme avec modération. On lui préfère dorénavant l'huile d'olive dont l'image est liée à celle d'une cuisine saine et légère, symbole d'un discours diététique et d'une alimentation méditerranéenne prônée par la médecine. Cette évolution s'est opérée dans une société qui ne perçoit plus les graisses comme ayant des valeurs gastronomiques; c'est là un profond changement qui influence encore aujourd'hui les pratiques culinaires.

55. L. S. R., L'Art de bien traiter, op.cit., p. 57. Dans l'édition originale, Paris, Jean Du Puis, 1674, p. 73. 
